\title{
Optical Anisotropy of Photo-Crosslinkable Polymer Film and Photoalignment Control of Nematic Liquid Crystals Using Nonpolarized Ultraviolet Irradiation
}

\author{
Nobuhiro Kawatsuki, ${ }^{\dagger}$ Tohei Yamamoto, and Hiroshi ONO* \\ Department of Applied Chemistry, Himeji Institute of Technology, \\ 2167 Shosha, Himeji 671-2201, Japan \\ * Department of Electrical Engineering, Nagaoka University of Technology, \\ 1603-1 Kamitomioka, Nagaoka 940-2188, Japan
}

(Received February 16, 1999)

\begin{abstract}
KEY WORDS Photochemistry / Cinnamic Ester / Photoalignment / Photo-Crosslinkable Polymer / Polymer Liquid Crystals
\end{abstract}

The photoalignment technique for liquid crystalline display (LCD) has received much attention in recent years because of its dust-free and photo-patternable process. ${ }^{1}$ Several kinds of photoreactive polymers for the photoalignment based on anisotropic photoisomerization or photo-crosslinking reactions have been reported. ${ }^{2-10}$ Nematic liquid crystal (LC) can be aligned homogeneously on these photoreacted polymer films which are usually exposed by a linearly polarized (LP) light. To obtain tilt angles for LC alignment using an LP light, slantwise irradiation is utilized for a material that aligns LC parallel to the electric vector $(\boldsymbol{E})$ of the incident polarized light. ${ }^{8,9}$ On the other hand, two-step irradiation is employed for a material that aligns LC perpendicular to $\boldsymbol{E}^{10}$ However, the use of LP light reduces the intensity of light sources to at least one-half of its initial value when using polarization components, and such devices are usually expensive.

Recently, Ichimura et al. first reported one-step slantwise nonpolarized irradiation on an azobenzenecontaining polymer film to induce three-dimensional orientation of the chromophore. ${ }^{11,12}$ In this kind of irradiation, the reorientation of the azobenzene chromophore is generated with a direction parallel to the incident light propagation, making it possible for nematic LC to be aligned with tilt angles on this film. ${ }^{11}$ This new technique proposes a new way for the photoalignment based on direction-selective photochemistry. ${ }^{11,12}$

From a practical point of view for the LCD industry, the thermal stability of the alignment layer is very important, and the alignment layer must be transparent in a visible region for the display devices. A photo-crosslinkable polymer system can offer high thermal stability because of its three-dimensional network, and several photo-crosslinkable polymers for the photoalignment have been investigated. ${ }^{4-10}$ However, all of these materials were irradiated by LP ultraviolet (UV) light to accomplish the LC alignment. The purpose of this paper is to describe an anisotropic photo-crosslinking reaction of a polymer film containing photo-crosslinkable 4-cinnamoyloxybiphenyl group by a slantwise nonpolarized UV irradiation, and a photoalign- ment control of nematic LC with tilt angles on the resultant film.

\section{EXPERIMENTAL}

The chemical structure of the photo-crosslinkable polymer 1 used in this study is shown in Figure 1. This polymer exhibited liquid crystalline phase between $144^{\circ} \mathrm{C}$ and $219^{\circ} \mathrm{C} .{ }^{13}$ The polymer was dissolved in methylene chloride $(0.5 \mathrm{wt} / \mathrm{wt} \%)$, and the solution was spin-coated on a quartz or $\mathrm{CaF}_{2}$ substrate, resulting in a $65-\mathrm{nm}$ thick film. The spin-coated film was transparent with no-optical anisotropy. We did not anneal the film before irradiation to avoid a formation of a liquid crystalline domain in the film. The film, which had no-absorption in a visible region, was exposed with a high-pressure $\mathrm{Hg}$-UV light source with a cut-filter under $290 \mathrm{~nm}$. The intensity was about $40 \mathrm{~mW} \mathrm{~cm}^{-2}$ at $313 \mathrm{~nm}$ in all cases. Slantwise nonpolarized UV irradiation was performed with irradiation angles $(\theta)$ among $35^{\circ}-55^{\circ}$ at room temperature. The progress of photoreaction was determined by FT-IR with monitoring the decrease of intensity of the $-\mathrm{C}=\mathrm{C}-$ vibration $\left(1637 \mathrm{~cm}^{-1}\right)$ of cinnamoyl group in $1 .{ }^{13}$ Polarized UV-VIS spectroscopy, and preparation and evaluation of a nematic LC cell were performed as the same method as described previously. ${ }^{9}$

\section{RESULTS AND DISCUSSION}

Figure 2 exhibits a schematic view of the light incidence and the change in absorption spectrum before and after slantwise photoirradiation for $3 \mathrm{~J} \mathrm{~cm}^{-2}$. The UV irradiation angle was $35^{\circ}$. After exposure, absorption decreased due to the $[2+2]$ photo-crosslinking reaction

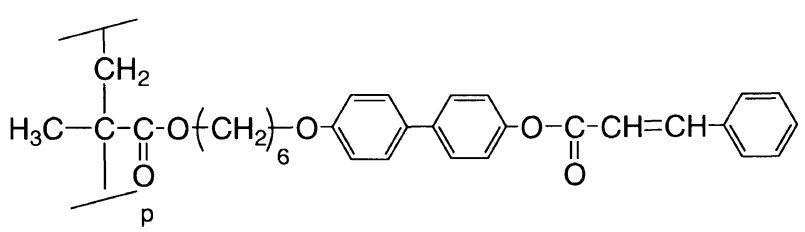

Figure 1. Chemical structure of photo-crosslinkable polymer $\mathbf{1}$

† To whom correspondence should be addressed (e-mail: kawatuki@ chem.eng.himeji-tech.ac.jp). 


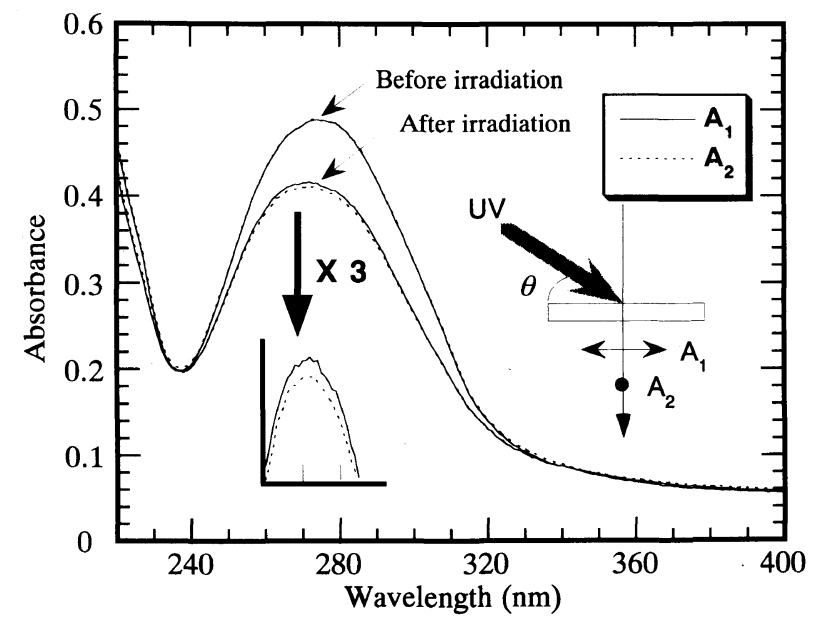

Figure 2. Schematic view of light incidences and absorption spectrum for the photo-crosslinkable polymer 1 film before and after slantwise nonpolarized UV exposure. The UV irradiation angle $\theta$ was $35^{\circ}$. $\mathrm{A}_{1}$ and $A_{2}$ mean electric vector direction of the measurement light. Film thickness was $65 \mathrm{~nm}$.

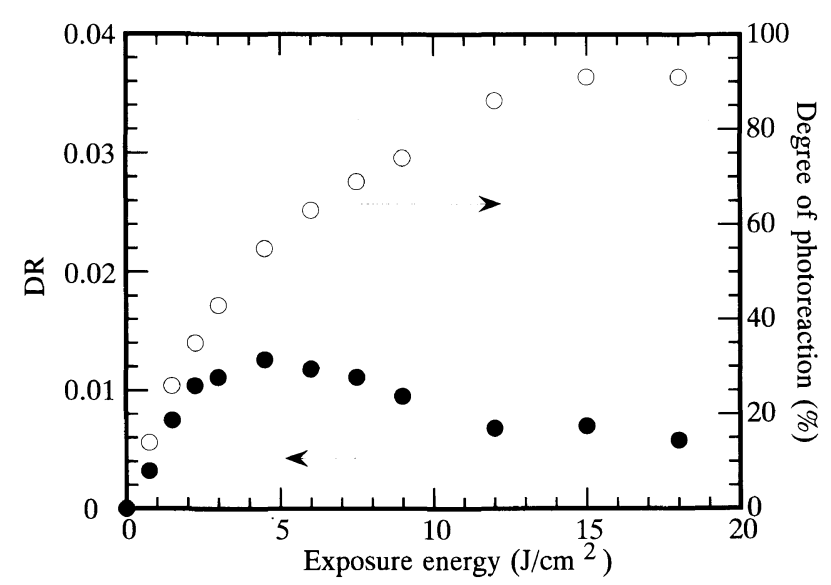

Figure 3. Dichroic ratio and degree of the photoreaction of a polymer 1 film as a function of exposure energy.

of a cinnamoyl group in the mesogenic group, ${ }^{13}$ and the absorbance of the $\mathrm{A}_{2}$ direction (perpendicular to the incident UV radiation) was smaller than that of the $A_{1}$ direction. It was reported that similar dichroism for an azobenzene-containing polymer film was induced by nonpolarized light exposure, and the dichroism was generated by $E-Z-E$ reorientation of the azobenzene chromophore. ${ }^{11,12}$ In contrast to the azobenzenecontaining polymer, reorientation of the mesogenic group could not occur for the polymer 1, indicating that the dichroism was initiated by the direction-selective photo-crosslinking reaction of the mesogenic group. In other words, the degree of the photo-crosslinking in the $\mathrm{A}_{2}$ direction was larger than that in the $\mathrm{A}_{1}$ direction. Figure 3 plots the dichroic ratio $\left(D R=\left(\mathrm{A}_{1}-\mathrm{A}_{2}\right) /\left(\mathrm{A}_{1}+\right.\right.$ $\left.A_{2}\right)$ ) and the degree of the photoreaction of the polymer film as a function of exposure energy. The $D R$ value increased until the degree of the reaction was $55 \%$, and decreased with further irradiation since the photocrosslinking of $\mathrm{A}_{1}$ direction could also occur. Furthermore, the dichroism diminished when the film was exposed from the opposite angle. This indicates that the photo-crosslinking reaction of this system does not follow

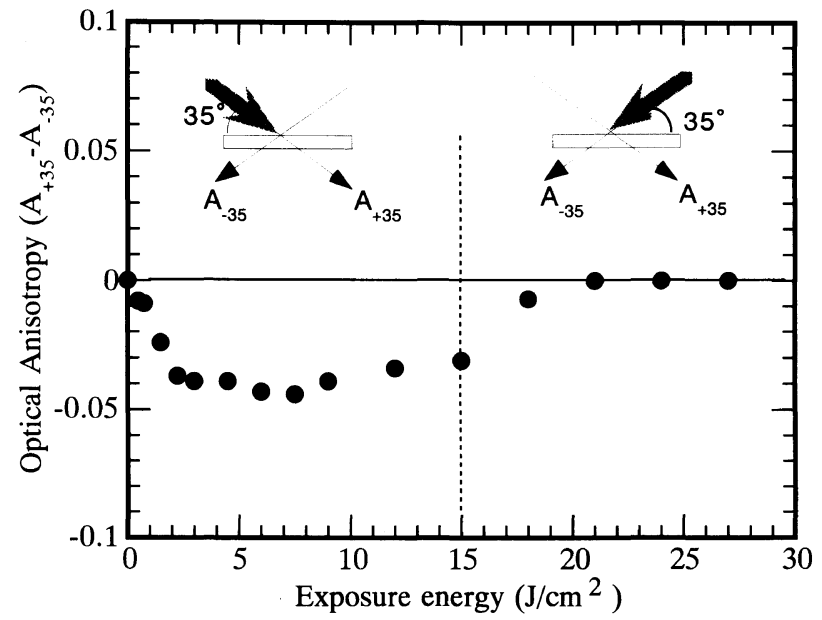

Figure 4. Change in the optical anisotropy of a polymer 1 film by irradiation of nonpolarized UV light. The incident angle of the light was $35^{\circ}$ at first and changed to $-35^{\circ}$ after $15 \mathrm{~J} \mathrm{~cm}^{-2}$ doses. The difference between the two absorbances $\left(A_{+35}-A_{-35}\right)$ at $272 \mathrm{~nm}$ was plotted as a function of the exposure energy. The thin arrows correspond to monitoring light, and the broad arrow exhibits the actinic UV light.

a reorientation of the mesogenic group. Therefore, the remaining small dichroism may be arisen from the non-photo-crosslinked mesogens with the direction parallel to the incident UV-light propagation.

This direction-selective photo-crosslinking reaction was confirmed by a slantwise-UV absorption measurement. The film was obliquely irradiated, followed by measuring absorbances $\left(A_{+35}\right.$ and $\left.A_{-35}\right)$ of the chromophore using monitoring lights with the same and the opposite incidence directions. Figure 4 plots the changes in the optical anisotropy of the film as a function of the exposure energy. The absorbance difference $\left(\Delta A=A_{+35}-A_{-35}\right)$ became negative after irradiation. This indicates that the mesogenic group in the parallel direction to the incident UV light propagation cannot photo-crosslink. In addition, the absolute value of $\Delta A$ of the polymer 1 film decreased with continuous irradiation, while $\Delta A$ did not become zero. Consequently, a certain amount of the mesogenic group parallel to the direction of the incident UV light propagation did not photoreact with continuous exposure, resulting in the three dimensional optical anisotropy of the film induced by the direction-selective photo-crosslinking reaction. In addition, the anisotropy did drop to zero when the UV light was exposed from the opposite direction since the remained mesogenic groups photo-crosslinked.

To investigate the alignment behavior of a nematic LC on the obliquely irradiated 1 film with non-polarized UV light, an anti-parallel LC-cell was fabricated, and the tilt angles were measured by the crystal rotation method. We reported that the alignment of nematic LC was achieved on an anisotropically photo-crosslinked polymer 1 film irradiated with LP-UV light, and that the direction of the non-photo-crosslinked mesogenic group determined the LC alignment direction. ${ }^{7}$ Although the optical anisotropy of the polymer 1 film induced by slantwise nonpolarized UV irradiation was very small, homogeneous alignment of a nematic LC with tilt angles could be generated when the exposure doses were more 


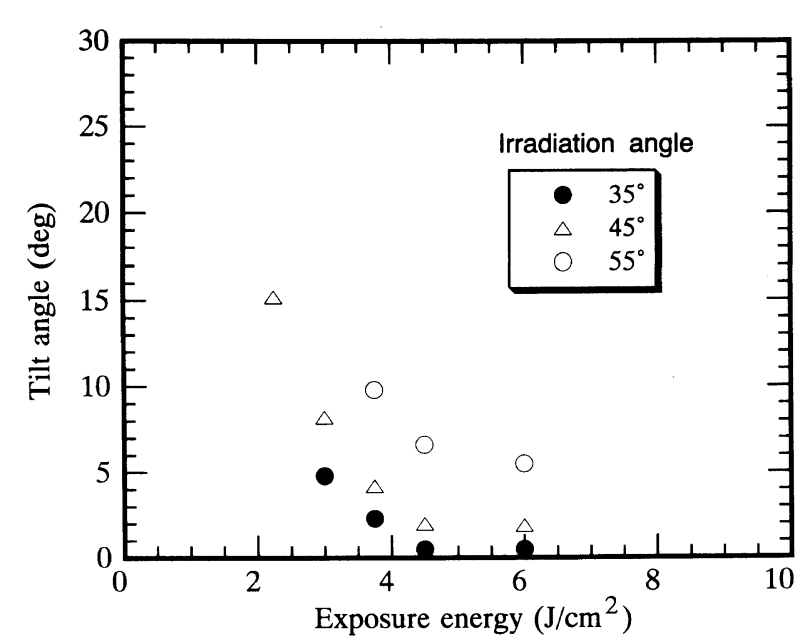

Figure 5. Tilt angles of a nematic LC (E7: Merck Japan) cell filled in an anti-parallel cell fabricated with two substrates coated with photo-crosslinked polymer 1 film as a function of exposure energy. The substrate was irradiated before cell fabrication. Incident irradiation angle: $0,35^{\circ} ; \triangle, 45^{\circ} ; \bigcirc, 55^{\circ}$.

than $2 \mathrm{~J} \mathrm{~cm}^{-2}$, and no defect was observed in the LC cell. It was confirmed that the tilting direction of $\mathrm{LC}$ molecules coincides with the incident direction of the UV light. As shown in Figure 5, tilt angles are dependent on the incident irradiation angles and exposure doses, becoming a saturated value. The saturated tilt angle was larger for the large irradiation angle than for the small one, suggesting that the non-photo-crosslinked mesogenic group remains oblique and the LC molecule aligns along these mesogens. In addition, tilt angles were smaller than the irradiation angle in all cases. We expect that the tilt angle of the non-photo-crosslinked mesogenic group in the film surface will not be equal to the incident UV-light propagation and is varied with the degree of exposure doses. However, it is difficult to obtain the reason for this small tilt angle of the nematic LC at present.

In summary, we investigated the slantwise nonpolarized UV photoreaction of photo-crosslinkable polymer film based on direction-selective photochemistry. ${ }^{11}$ Because of the direction-selective photocrosslinking reaction, three-dimensional optical anisotropy of the film was generated. The homogeneous alignment of nematic LC with tilt angles on the resultant films could be controlled by changing the irradiation angle and exposure doses. We anticipate that this polymer system will be useful to the LCD industry as the photoalignment layer.

\section{REFERENCES}

1. K. Ichimura, "Polymers as Electronical and Photooptical Active Media," V. P. Shibaev, Ed., Springer, Berlin, 1996, p 138.

2. K. Ichimura, Y. Suzuki, T. Seki, A. Hosoki, and K. Aoki, Langmuir, 4, 1214 (1988).

3. W. M. Gibbons, P. J. Shannon, S.-T. Sun, and B. J. Swetlin, Nature (London), 351, 49 (1991).

4. M. Schadt, K. Schmitt, V. Kozinkov, and V. Chigrinov, Jpn. J. Appl. Phys., 31, 2155 (1992).

5. K. Ichimura, Y. Akita, H. Akiyama, K. Kudo and Y. Hayashi, Macromolecules, 30, 903 (1997).

6. N. Kawatsuki, H. Takatsuka, T. Yamamoto, and H. Ono, Jpn. J. Appl. Phys., 36, 6464 (1997).

7. N. Kawatsuki, K. Takatani, T. Yamamoto, and H. Ono, Polym. J., 30, 946 (1998).

8. M. Schadt, H. Seiberle, and A. Schuster, Nature (London), 381, 212 (1996).

9. N. Kawatsuki, H. Ono, H. Takatsuka, T. Yamamoto, and O Sangen, Macromolecules, 30, 6680 (1997).

10. Y. Iimura, T. Saitoh, S. Kobayashi, and T. Hashimoto, $J$. Photopolym. Sci. Tech., 2, 257 (1995).

11. K. Ichimura, S. Morino, and H. Akiyama, Appl. Phys. Lett., 73, 921 (1998).

12. K. Ichimura, M. Han, and S. Morino, Chem. Lett., 85 (1999).

13. N. Kawatsuki, S. Sakashita, K. Takatani, T. Yamamoto, and O. Sangen, Macromol. Chem. Phys., 197, 1919 (1996). 